\section{Correlação entre níveis de iodo na urina e alterações anatomopatológicas em tireoides}

\author{
Correlation between iodine urinary levels and \\ pathological changes in thyroid glands
}

Paulo César Ribeiro Boasquevisque', Robson Dettman Jarske ${ }^{1,2}$, Cleia Coelho Dias', Isabela Passos Pereira Quintaes', Maria Carmen Lopes Ferreira Silva Santos ${ }^{1,2}$, Carlos Musso',2

\begin{abstract}
RESUMO
Objetivos: Determinar o aporte nutricional de iodo na população estudada e correlacionar os níveis de iodo encontrados em amostras casuais de urina (iodúria) com alterações anatomopatológicas observadas nas tireoides dessa população. Materiais e métodos: Determinou-se a iodúria em 30 amostras casuais de urina e realizou-se o estudo anatomopatológico de 55 tireoides colhidas de cadáveres que deram entrada no Departamento Médico Legal de Vitória, Espírito Santo, Brasil, no período de maio a agosto de 2011. Resultados: Em 29 amostras de urina $(96,7 \%)$ encontrou-se iodúria acima do limite máximo preconizado pela Organização Mundial da Saúde (OMS) de $300 \mu \mathrm{g} / \mathrm{L}$. Em 14 tireoides $(25,5 \%)$ foi identificada a presença de quadros histológicos compatíveis com tireoidite. Níveis mais elevados de iodo na urina foram observados no sexo feminino e nos casos de tireoides com presença de achados inflamatórios (tireoidites). Conclusões: Podemos concluir que na população estudada houve um excesso de iodo em seu aporte nutricional e uma maior incidência de quadros inflamatórios tireoidianos. Arq Bras Endocrinol Metab. 2013;57(9):727-32
\end{abstract}

\section{Descritores}

lodo; tireoide; tireoidite; iodúria; excesso de iodo

\begin{abstract}
Objectives: To determine iodine nutrition in the population and to correlate levels of iodine found in random samples of urine with pathological changes observed in thyroids collected in this population. Materials and methods: Urinary iodine was determined in 30 random samples of urine and the pathological study was carried out in 55 thyroid glands from corpses received by the Department of Forensic Medicine of Vitória, Espírito Santo, Brazil from May to August 2011. Results: In 29 urine samples $(96.7 \%)$ urinary iodine was above the maximum limit recommended by the World Health Organization (WHO), of $300 \mathrm{mg} / \mathrm{L}$. Fourteen thyroids (25.5\%) showed the presence of histological changes compatible with thyroiditis. Higher levels of iodine in urine were observed in females and in of thyroid that showed inflammation (thyroiditis). Conclusions: We conclude that, in this population, there is excess iodine intake, and greater incidence of inflammatory thyroid disease. Arq Bras Endocrinol Metab. 2013;57(9):727-32
\end{abstract}

Keywords

lodine; thyroid; thyroiditis; urinary iodine; excess iodine
1 Universidade Federal do Espírito Santo (UFES), Vitória, ES, Brasil ${ }^{2}$ Universidade Vila Velha (UVV-ES), Vila Velha, ES, Brasil
Correspondência para: Paulo César Ribeiro Boasquevisque Rua Castelo Branco, 139/902, Praia da Costa 29101-485 - Vila Velha, ES, Brasil pauloboasq@terra.com.br

Recebido em 19/Mai/2013 Aceito em 28/Jun/2013

\section{INTRODUÇÃO}

$\mathrm{O}$ iodo é um oligoelemento essencial ao funcionamento da glândula tireoide e a dieta alimentar representa sua fonte exclusiva para o organismo humano. É encontrado principalmente em peixes, mariscos, frutos do mar, ovos, leite e derivados, entre outros. $\mathrm{O}$ sal comum de cozinha é importante fonte nutricio- nal de iodo nos países que adotam a sua adição ao sal, como ocorre no Brasil $(1,2)$.

A concentração de iodo no sal de cozinha no Brasil tem sofrido alterações ao longo dos anos. De 1998 a 2003 essa adição foi de 40 a 100 mg de iodo por quilograma de sal. Entre 2003 até recentemente vigorou a Resolução da Diretoria Colegiada da Agência Nacional 
de Vigilância Sanitária (RDC/Anvisa) 130/2003 considerando próprio para o consumo o sal com teor de 20 a $60 \mathrm{mg}$ de iodo por quilograma do produto $(3,4)$. Em 24/4/2013 foi editada a RDC/Anvisa 23/2013 alterando essa concentração para 15 a $45 \mathrm{mg}$ de iodo $/ \mathrm{kg}$ sal de cozinha (5).

Ações de monitoramento do estado nutricional do iodo na população são necessárias quando a iodação do sal é adotada, uma vez que tanto o déficit quanto o excesso desse micronutriente criam riscos potenciais à saúde $(6,7)$.

O excesso de iodo é considerado fator de risco para o aparecimento de alterações funcionais da tireoide como hipertireoidismo e tireoidite crônica autoimune de Hashimoto (8). Variações nutricionais de iodo têm sido relacionadas também com a ocorrência de neoplasias malignas da tireoide (9-11).

Os indicadores usados habitualmente para se avaliar o aporte nutricional de iodo de uma população são a concentração de iodo adicionado ao sal de cozinha, os níveis de iodo eliminados na urina e o volume tireoidiano (10-12).

A determinação dos níveis de iodúria é considerada atualmente o marcador bioquímico mais fidedigno do aporte nutricional do iodo em estudos epidemiológicos (9). Segundo a Organização Mundial da Saúde (OMS), em indivíduos com ingesta adequada de iodo devem ser eliminados diariamente entre 100 e $300 \mu \mathrm{g}$ de iodo por litro de urina $(9,13,14)$.

Em estudos populacionais, pelas dificuldades técnicas de se colher o volume urinário de 24 horas, admite-se a coleta de amostra casual de urina para essa finalidade, o que de acordo com a OMS reflete o perfil epidemiológico nutricional do iodo na população (9$13)$.

$\mathrm{O}$ fato de que o brasileiro ingere mais sal do que o recomendado e consequentemente mais iodo merece atenção. A recomendação atual da OMS é de que se consuma o equivalente a cinco gramas de sal por dia. $\mathrm{O}$ Instituto Brasileiro de Geografia e Estatística (IBGE) relata que o consumo de sal per capita no Brasil é de 10 g/dia e a Associação Brasileira de Extratores e Refinadores de Sal refere ser de 14 a $16 \mathrm{~g} /$ dia $(4,15)$.

Trabalho realizado na Universidade Federal do Espírito Santo identificou que o consumo de sal per capita diário em Vitória, capital do estado do Espírito Santo, é em média de 12,5 gramas (16,17).

Os objetivos do nosso estudo foram determinar o aporte nutricional de iodo na população estudada e in- vestigar a associação entre a concentração dos níveis de iodo em amostras casuais de urina com alterações histopatológicas em tireoides obtidas de cadáveres de morte recente de até 24 horas.

\section{MATERIAIS E MÉTODOS}

Este Estudo recebeu aprovação do Comitê de Ética em Pesquisa em Seres Humanos da Universidade Vila Velha, Espírito Santo, em maio 2011 sob o número $08 / 2011$.

No período de maio a agosto 2011 , foram captadas 55 tireoides de cadáveres de morte recente de até 24 horas que deram entrada no Departamento Médico Legal de Vitória, Espírito Santo, Brasil, sendo 18 tireoides de cadáveres femininos e 37 de cadáveres masculinos. Em 30 casos (17 femininos/13 masculinos), foi possível a coleta de amostra casual de urina, realizando-se nesses a análise da iodúria. Todas as captações foram autorizadas por parentes de $1^{\circ}$ grau das vítimas por meio de Termo de Consentimento Livre e Esclarecido.

Foram incluídos no estudo cadáveres de pessoas que residiram no estado do Espírito Santo pelo menos nos últimos cinco anos de suas vidas, que não tinham sido submetidos a tratamento clínico ou cirúrgico de doenças da tireoide ou uso terapêutico do iodo, que não eram portadores de doenças renais conhecidas ou fizessem uso de medicamentos contendo amiodarona, furosemida ou derivados do lítio por caracterizarem situações que alteram a dosagem do iodo na urina. $\mathrm{O}$ histórico médico pregresso do cadáver foi pesquisado por intermédio de questionamento direto aos familiares que procederam ao reconhecimento legal do cadáver.

As glândulas tireoides foram retiradas inteiras em seu plano de clivagem junto a traqueia e acondicionadas em frascos transparentes de polietileno contendo solução de formol a 10\%. Após convenientemente fixadas, foram pesadas em balança de precisão na casa centesimal e tomados seus volumes.

Procedida a abertura da cavidade toracoabdominal e exposta a bexiga urinária, foi realizada punção transparietal em parede anterossuperior da bexiga utilizando-se material descartável composto de seringa de plástico transparente de volume de $20 \mathrm{ml}$ acoplada a agulha 40 x 12, realizando-se aspiração manual contínua com a finalidade de se obter amostra casual de urina do cadáver, que uma vez obtida foi transferida para frasco plástico transparente de $50 \mathrm{ml}$ de volume. 
As amostras de urina coletadas foram acondicionadas sob refrigeração a $-20^{\circ} \mathrm{C}$ até o momento de sua análise, que foi realizada por meio do Laboratório Carlos Chagas de Vitória, Espírito Santo. O método utilizado para a determinação da iodúria foi o da Potenciometria com Eletrodo Íon Seletivo realizado em aparelho da marca Crison ${ }^{\circledR}$, e os resultados foram emitidos em microgramas de iodo por litro de urina.

$\mathrm{O}$ volume da glândula tireoide foi aferido utilizando-se o princípio de Arquimedes para a Lei da Hidrostática. Assim, cada glândula isoladamente foi totalmente submersa em recipiente transparente graduado em mililitros contendo $150 \mathrm{ml}$ de água. O volume da tireoide correspondeu à diferença entre o volume do meio líquido após a imersão completa da glândula neste meio e o volume inicial do meio líquido.

As tireoides fixadas em solução de formol a $10 \%$ foram retiradas do fixador, acondicionadas individualmente em sacolas transparentes com zíper e submetidas a exame de ultrassonografia a temperatura ambiente com aparelho da marca Esaote modelo MyLab 70 X Vision equipado com transdutor linear de $18 \mathrm{MHz}$.

Com a finalidade de anular as variações de ganho de brilho com interferência na determinação da ecogenicidade das tireoides, todas as imagens foram obtidas com a mesma intensidade de ganho de brilho de $64 \%$ e registradas pelo mesmo ultrassonografista. Cortes axiais em toda a extensão da glândula foram realizados com registro das lesões observadas e uma imagem de cada lobo tireoidiano foi gravada em arquivo digital e transferida para o computador para que se procedesse a determinação da ecogenicidade da glândula que foi quantificada utilizando-se o software Image J.

Em cada lobo tireoidiano foram delimitadas aleatoriamente três áreas para estudo e tomados os seu valores no histograma. O resultado final do histograma de cada tireoide representou a média dessas seis medidas. Da mesma forma, tomaram-se seis medidas do músculo esternocleidomastóideo e o resultado de seu histograma representou a média dessas medidas.
Visando manter a integridade das tireoides para estudos posteriores, elas foram biopsiadas na área central de cada lobo com punch de 5 milímetros, obtendo-se dois fragmentos cilíndricos de toda a espessura dos lobos tireoidianos que foram processados para inclusão em parafina, dos quais foram obtidos cortes histológicos corados pelo método da Hematoxilina e Eosina.

As análises histológicas foram realizadas pelo mesmo examinador de forma seriada e cega com registro de todas as alterações tireoidianas observadas. Foram analisados o aspecto dos folículos e do coloide, a presença de infiltrado inflamatório e outras alterações. Considerou-se como foco inflamatório a presença de aglomerado linfoide com mais de 50 células, sendo especificadas a sua localização, distribuição e intensidade.

Neste estudo, as variáveis foram tratadas estatisticamente utilizando-se os cálculos não paramétricos Teste do Qui-quadrado, Teste de Mann-Whitney e Coeficiente de Correlação de Spearman. Determinaram-se a mediana, média aritmética, desvio-padrão, valor mínimo e valor máximo dos dados, números absolutos e cálculos percentuais. Os programas estatísticos mais utilizados foram Microsoft Excel 2010, SPSS (IBM Corporate) release 19.0 e EpiInfo 3.5.3.

\section{RESULTADOS}

Foram analisados estatisticamente os dados obtidos de 55 tireoides e 30 amostras casuais de urina (Tabela 1 ).

Das 30 amostras casuais de urina submetidas à determinação da iodúria, 29 delas $(96,7 \%)$ apresentaram valores acima de $300 \mu \mathrm{g} / \mathrm{L}$ e em somente 1 amostra $(3,3 \%)$ o valor estava dentro dos limites considerados normais pela OMS.

Das amostras casuais de urina, 21 amostras (70\%) foram obtidas em tempo de até 12 horas post-mortem e 9 delas (30\%) foram coletadas em tempo que se situou entre 12 e 24 horas post-mortem, sendo que, para o grupo de até 12 horas post-mortem, a mediana da idade foi de 31 anos e a mediana dos níveis de iodúria foi de

Tabela 1. Estatísticas descritivas das variáveis estudadas

\begin{tabular}{lcccccc}
\hline Variáveis & $\mathbf{n}$ & Menor valor & Maior valor & Mediana & Média & Desvio-padrão \\
\hline Idade (anos) & 55 & 15,00 & 94,00 & 31,00 & 37,64 & 20,82 \\
Níveis de iodúria $(\mu \mathrm{g} / \mathrm{L})$ & 30 & 162,00 & $3.250,00$ & 436,50 & 648,56 & 591,91 \\
Peso tireoides $(\mathrm{g})$ & 55 & 6,15 & 33,87 & 14,02 & 15,42 & 5,96 \\
Volume tireoides $(\mathrm{ml})$ & 55 & 6,00 & 29,00 & 13,00 & 13,69 & 4,43 \\
Ecogenicidade tireoides & 55 & 31,55 & 64,82 & 44,70 & 45,82 & 6,24 \\
\hline
\end{tabular}


$470 \mu \mathrm{g} / \mathrm{L}$ e, para o grupo de 12 a 24 horas post-mortem, foi de 30 anos e $382 \mu \mathrm{g} / \mathrm{L}$, respectivamente.

Para a comparação entre os dois grupos, foi utilizado o teste de Mann-Whitney, constatando-se não ter havido diferença estatisticamente significante entre os grupos em relação aos resultados das medianas dos níveis de iodúria $(\mathrm{p}=0,057)$.

Em seis das tireoides $(10,9 \%)$ foram encontradas lesões focais (cistos ou nódulos) no exame de ultrassonografia.

As análises histológicas foram agrupadas quanto à presença de tireoidite e de outras alterações histopatológicas e, em somente nove tireoides $(16,3 \%)$, não foram observadas alterações dignas de nota (Tabela 2 ).

Tabela 2. Alterações histopatológicas encontradas nas tireoides

\begin{tabular}{lcc}
\hline Alterações histopatológicas & $\mathbf{n}$ & \% \\
\hline Tireoidites & 14 & 25,5 \\
Coloide cristaloide & 41 & 74,5 \\
Invasão macrofágica do coloide & 9 & 16,3 \\
Hiperplasia papilar intrafolicular & 2 & 3,6 \\
Hipotrofia dos folículos & 6 & 10,9 \\
Grande variação do tamanho dos folículos & 4 & 7,2 \\
Alterações do estroma tireoidiano* & 3 & 5,4 \\
Bócio nodular hiperplásico & 3 & 5,4 \\
Bócio coloide & 1 & 1,8 \\
Bócio adenomatoso multinodular & 1 & 1,8 \\
Outras alterações tireoidianas & 8 & 14,5 \\
Total & 55 & 100,00 \\
\hline
\end{tabular}

* Inclui fibrose ou esclerose do estroma interfolicular. ** Inclui presença de paratireoide intratireoidiana, folículos basófilos, metaplasia oxifilica, corpo último branquial, cisto coloide e epitélio folicular fino aplanado.

Achados histológicos compatíveis com tireoidite (foco inflamatório, infiltração inflamatória difusa ou nódulos linfoides) foram encontrados em 14 tireoides (25,5\%).

As variáveis foram testadas entre si aplicando-se o Teste de Mann-Whitney (Tabelas 3 e 4) e o Coeficiente de Correlação de Spearman (Tabela 5).

Tabela 3. Comparação da variável sexo com níveis de iodúria, peso, volume e ecogenicidade das tireoides

\begin{tabular}{|c|c|c|c|}
\hline Variáveis & Sexo & Mediana & p-valor \\
\hline \multirow[t]{2}{*}{ Níveis de iodúria ( $\mu \mathrm{g} / \mathrm{L})$} & Masculino & 415,00 & $0,027^{*}$ \\
\hline & Feminino & 550,00 & \\
\hline \multirow[t]{2}{*}{ Peso tireoides (g) } & Masculino & 15,50 & $0,021^{*}$ \\
\hline & Feminino & 12,60 & \\
\hline \multirow[t]{2}{*}{ Volume tireoides (mL) } & Masculino & 14,00 & $0,043^{*}$ \\
\hline & Feminino & 12,00 & \\
\hline \multirow[t]{2}{*}{ Ecogenicidade tireoides } & Masculino & 44,31 & 0,379 \\
\hline & Feminino & 46,99 & \\
\hline
\end{tabular}

*Estatisticamente significativo.
Tabela 4. Comparação entre níveis de iodúria, presença de tireoidite e de coloide cristaloide

\begin{tabular}{lccc}
\hline Variáveis & & $\begin{array}{c}\text { Níveis de } \\
\text { iodúria** }^{\star *}\end{array}$ & p-valor \\
\hline Tireoidite & Presente & 530,00 & $0,032^{\star}$ \\
& Ausente & 413,50 & \\
Coloide cristaloide & Sim & 460,00 & 0,334 \\
& Não & 415,00 & \\
\hline
\end{tabular}

* Estatisticamente significativo; ** Mediana.

Tabela 5. Correlação entre níveis de iodúria com peso, volume e ecogenicidade das tireoides

\begin{tabular}{lcc}
\hline Níveis de iodúria & $\begin{array}{c}\text { Coeficiente de } \\
\text { correlação }\end{array}$ & p-valor \\
\hline Peso tireoides $(\mathrm{g})$ & $-0,378$ & $0,043^{*}$ \\
Volume tireoides $(\mathrm{mL})$ & $-0,353$ & 0,060 \\
Ecogenicidade tireoides & 0,209 & 0,276 \\
\hline
\end{tabular}

* Estatisticamente significativo.

\section{DISCUSSÃO}

O estudo em cadáveres permite a coleta de toda a glândula tireoide e a análise direta de seus parâmetros anatômicos e histológicos, reduzindo significativamente interferências relacionadas a sensibilidade e especificidade dos métodos habitualmente utilizados para esses fins, tais como a ultrassonografia. Entretanto, apresenta a impossibilidade de se determinar a presença de anticorpos antitireoidianos, dosar os níveis dos hormônios tireoidianos ou obter a coleta da urina de 24 horas.

Essas impossibilidades não interferem de forma relevante, uma vez que as alterações histológicas precedem o aparecimento dos anticorpos antitireoidianos, e a coleta de amostra casual de urina para determinação da iodúria está amplamente sedimentada como indicador de aporte nutricional do iodo $(9-11,13)$.

Em nosso material de análise, a área examinada se aproximou de $1,5 \mathrm{~cm}^{2} \mathrm{e}$, considerando-se que a unidade funcional da tireoide é o folículo, as amostras examinadas incluíram centenas deles, tornando-as suficientes para a avaliação de inflamação nas amostras de tecido removido das tireoides.

Semelhante ao descrito na literatura, observamos em nosso estudo um índice de $25,5 \%$ de quadros histológicos de tireoidites. A nossa série foi composta de pacientes assintomáticos do ponto de vista de doenças da tireoide. É possível que essa incidência maior de casos de tireoidites em estudos de necropsia deva-se ao fato de que as alterações histológicas precedam as manifestações clínicas que permitam supor seu diagnóstico (18-20). 
Em nossa série, níveis mais elevados de iodo na urina foram observados nos casos com presença de tireoidite (Tabela 4).

As principais hipóteses aceitas indicam que o excesso de iodo promove uma maior iodinização da tireoglobulina conferindo-lhe um potencial imunogênico elevado, estimulando a produção de anticorpos antitireoglobulina (anti-Tg). Além disso, radicais livres ligam-se ao iodo em excesso na tireoide originando compostos lesivos à glândula, resultando na liberação de fatores quimiotáxicos, produção de anticorpos anti-TPO, anti-Tg e anti-NIS e consequente infiltração linfocitária na glândula tireoide (18).

Níveis mais elevados de iodúria foram observados em nosso estudo em mulheres. Não encontramos na literatura descrição que explique a predominância de níveis mais elevados de iodo na urina no sexo feminino, que foi um dado relevante encontrado por nós (Tabela 3 ).

A presença do aspecto cristaloide no coloide folicular foi observada em 74,5\% dos casos, variando de raros a frequentes. Esse achado pode ser atribuído ao fenômeno de coagulação da tireoglobulina e precipitação de grumos em meio ao coloide, não se conhecendo até o momento o mecanismo desse processo. Esse fenômeno não sugere ser de origem artefatual uma vez que é também encontrado com frequência em esfregaços obtidos por punção com agulha fina.

Esse aspecto coloidal cristaloide pode ser encontrado em tireoides normais e tende a aumentar com a idade. Igualmente foi observado em quantidade maior em portadores de tireoidite subaguda ou insuficiência renal submetidos à diálise. Em estudos de necropsia, a presença de cristais no coloide folicular foi observada em até $85 \%$ das tireoides examinadas $(21)$.

Apesar da alta frequência observada, não foi demonstrada correlação estatisticamente significante entre o coloide de aspecto cristaloide com as demais variáveis estudadas, inclusive quando comparadas com os níveis de iodúria (Tabela 4 ).

O volume e o peso tireoidianos apresentaram correlação estatisticamente significante com a variável sexo, e os homens apresentaram valores maiores em comparação com as mulheres (Tabela 4).

O cruzamento das variáveis níveis de iodúria e peso da tireoide apresentou correlação estatisticamente significante mas inversamente proporcional, ou seja, quanto menor o peso da glândula, maior os níveis de iodo na urina. Essa correlação, embora estatisticamente significativa, é de força baixa e negativa, ou seja, apresenta coeficiente de correlação muito baixo (Tabela 5 ).
Não houve diferença estatisticamente significativa quando comparados os valores dos histogramas da ecogenicidade das tireoides obtidas ao ultrassom com todas as demais variáveis. A ecogenicidade da tireoide expressa a interface celular-coloidal dominante da tireoide, que representa o fator essencial na reflexão do som, e sua ecogenicidade é normalmente superior à da musculatura cervical. Em nosso estudo, todas as glândulas tireoides apresentaram ecogenicidade menor do que a observada no músculo esternocleidomastóideo. A hipoecogenicidade constitui um padrão ultrassonográfico que costuma acompanhar os quadros de tireoidites (19).

É possível que essa hipoecogenicidade da tireoide observada em todos os cadáveres no grupo analisado, mesmo naqueles em que não se identificaram quadros de tireoidite, possa ter relação com a perda da congestão vascular sanguínea no cadáver. Entretanto, essa hipótese carece de confirmação.

\section{CONCLUSÃO}

Os resultados obtidos em nosso estudo sugerem haver uma ingesta excessiva de iodo pela população, particularmente pelo excesso de consumo de sal iodado eventualmente associado a hábitos alimentares que incluam outras fontes dietéticas ricas em iodo. Esse excesso de iodo reflete-se em alterações da glândula tireoide em graus variados compatíveis com a presença de tireoidite.

Estudos futuros com amostragens mais amplas são necessários para monitorar o padrão nutricional de iodo no estado do Espírito Santo e identificar seus reflexos na população em geral.

De fato, para uma população com a característica de ingerir sal de cozinha em quantidade bastante elevada como tem demonstrado ser a população brasileira, a adição de qualquer micronutriente a esse sal deve tornar-se motivo de atenção para a Saúde Pública. No caso específico do iodo, está de igual maneira, sendo ingerido em quantidades excessivas com os riscos inerentes a essa condição.

A redução da concentração dos níveis de iodo adicionado ao sal de cozinha no Brasil, como determinado recentemente pela Anvisa com a promulgação da RDC $23 / 2013$, evidencia que o excessivo aporte nutricional de iodo observado na população brasileira e sua correlação com o aumento dos casos de doenças da tireoide, como demonstrado pelos estudos epidemiológicos, consolidaram-se como um problema de Saúde Pública. 
A monitorização permanente do consumo de iodo pela população associada a programas educacionais que visem à redução efetiva do consumo de sal de cozinha no Brasil constitui ações fundamentais para a preservação da saúde do brasileiro.

Agradecimentos: a todos os familiares das pessoas falecidas que tiveram suas participações em nosso estudo por eles autorizadas, que, mesmo no momento de dor, demonstraram grandeza de espírito, permitindo que seus entes queridos oferecessem à sociedade uma última e grande contribuição do ponto de vista científico.

Declaração de financiamento, conflitos de interesse e quaisquer subsídios ou bolsas de apoio recebidos para a realização do trabalho: este artigo original é parte da dissertação de mestrado do Programa de Pós-Graduação em Ciências Farmacêuticas da Universidade Vila Velha (UVV-ES) com defesa de Dissertação apresentada em 8/3/2012 na cidade de Vila Velha, Espírito Santo. Este trabalho foi custeado com recursos próprios do autor principal. Os autores declaram não haver conflito de interesses com quaisquer instituições.

\section{REFERÊNCIAS}

1. Medeiros-Neto G. The salt iodization program in Brazil: a medical and political conundrum. IDD News. 2000;16(2):31-2.

2. Medeiros-Neto G. lodine nutrition in Brazil: where do we stand? Arq Bras Endocrinol Metab. 2009;53(4):470-4.

3. Brasil. Ministério da Saúde, Instituto Brasileiro de Geografia e Estatística e Ministério do Planejamento, Orçamento e Gestão. Pesquisa de Orçamentos Familiares 2002-2003. Antropometria e análise do estado nutricional de crianças e adolescentes no Brasil; 2006.

4. Brasil. Ministério da Saúde, Ministério da Ciência e Tecnologia, FINEP, DECIT. Termo de referência da pesquisa nacional para avaliação do impacto da iodação do sal. 2006.

5. Brasil. Agência Nacional de Vigilância Sanitária - Anvisa. Disponível em: http:// www.anvisa.gov.br. Acesso em: 29 Abr, 2013.

6. Esteves RZ, Kasamatsu TS, Kunii IS, Furuzawa GK, Vieira $\mathrm{JGH}$, Maciel RMB. Desenvolvimento de um método para a determinação da iodúria e sua aplicação na excreção urinária de iodo em escolares brasileiros. Arq Bras Endocrinol Metabol. 2007;51(9):1477-84.
7. Pretell EA, Delange F, Hostatec U, Corigliano S, Barreda L, Higa $A M$, et al. lodine improves in Latin America. Thyroid. 2004;14(8):590-5999.

8. Camargo RYA, Tomimori EK, Neves SC, Knobel M, Medeiros-Neto $\mathrm{G}$. Prevalence of chronic autoimmune thyroiditis in the urban area neighboring a petrochemical complex and a control area in São Paulo, Brazil. Clinics. 2006;61:307-12.

9. Delange $F$, de Benoist B, Bürgi $H$; the ICCIDD Working Group. Determining median urinary iodine concentration that indicates adequate iodine intake at population level. Bulletin of the World Health Organization. 2002;80(8):633-6.

10. Duarte GC, Tomimori EK, Boriolli RA, Ferreira JE, Catarino RM, Camargo RYA, et al. Avaliação ultra-sonográfica da tireoide e determinação da iodúria em escolares de diferentes regiões do Estado de São Paulo. Arq Bras Endocrinol Metab. 2004;48(6):842-8.

11. Duarte GC. Avaliação ultrassonográfica da tireoide, excreção urinária de iodo em escolares de 6 a 14 anos e grau de iodação do sal, em diferentes regiões do estado de São Paulo [tese]. São Paulo; 2007.

12. Rossi AC, Tomimori E, Camargo R, Medeiros-Neto G. Searching for iodine deficiency disorders in schoolchildren from Brazil: the Thyromobil project. Thyroid. 2001;11:661-3.

13. De Benoist B, Mclean E, Andersson M, Rogers L. lodine deficiency in 2007: global progress since 2003. Food Nutrit Bull. 2008;29(3):195-202.

14. World Health Organization. Recommended iodine levels in salt and guidelines for monitoring their adequacy and effectiveness. Fifth Report on World Nutrition, WHO 2004, March.

15. Agência Nacional de Vigilância Sanitária - Anvisa. Programa nacional garante qualidade do sal consumido no País. Rev Saúde Pública. 2004;38(4):611-2.

16. Molina MCB. Hipertensão arterial e fatores nutricionais: um estudo de base populacional no município de Vitória/ES [Tese]. Vitória: Universidade Federal do Espírito Santo; 2002.

17. Molina MDC, Cunha RS, Herkenhoff LF, Mill JG. Hipertensão arterial e consumo de sal em população urbana. Rev Saúde Pública. 2003;37(6):743-50.

18. Davies TF. Pathogenesis of Hashimoto's thyroiditis (chronic autoimmune thyroiditis). [CD-ROM]. Waltham: Up to date Desktop 19.1 Inc; 2011.

19. Höfling DB, Cerri GG, Juliano AG, Marui S, Chammas MC. Importância da ecogenicidade da tireóide no diagnóstico da tireoidite crônica autoimune. Radiol Bras. 2008;41(6):409-17.

20. Sgarbi JA, Maciel RM. Patogênese das doenças tiroideanas autoimunes. Arq Bras Endocrinol Metab. 2004;53(1):5-14.

21. Carcangiu LM. Thyroid. In: Stacey E. Mills, editor. Histology for pathologists, 3rd ed. Philadelphia: Lippincott Williams \& Wilkins; 2007. p. 1137. 